\title{
Clinicopathological profile of children evaluated for suspected tuberculosis in a rural medical college hospital
}

\author{
Padma Kalyanaraman', Sumathi $\mathbf{S}^{2}$ \\ From ${ }^{1}$ Associate Professor, Department of Pediatrics, ${ }^{2}$ Professor, Department of Pathology, Melmaruvathur Adhiparasakthi Institute of Medical \\ Sciences and Research, Melmaruvathur, Tamil Nadu, India
}

Correspondence to: Padma Kalyanaraman, A2 New Doctors Quarters, Melmaruvathur - 603 319, Tamil Nadu, India. E-mail: drpadmakalyan@yahoo.co.in

Received - 14 April 2019

Initial Review - 07 May 2019

Accepted - 26 June 2019

\begin{abstract}
Background: Tuberculosis (TB) is a major public health problem all over the world and India accounts for nearly one-third of the world TB burden. Pediatric TB has been estimated to account for approximately $20-40 \%$ of the cases in India. However, the diagnosis of childhood TB remains a challenge and is a major cause of concern in the eradication of TB. Objectives: The objectives of this study were to study the various clinical features and laboratory parameters in children suspected to have TB and to find out the most useful parameter to diagnose TB in children. Materials and Methods: This was a retrospective cross-sectional study conducted in a rural medical college hospital during the year 2017. Children up to the age of 14 years with a clinical diagnosis of suspicious TB were included in the study and the confirmation of TB was evaluated. All demographic data, contact history, symptoms, clinical findings, and laboratory parameters were retrieved from the medical records department and analyzed. Results: A total of 102 children were included in the present study and the predominant age group affected was between 5 and 10 years $(30.4 \%)$ with male predominance $(55 \%)$. The predominant clinical presentation was cervical lymphadenopathy $(\mathrm{n}=46 ; 45 \%)$ followed by contact history of contact with TB ( $\mathrm{n}=34 ; 33.3 \%)$. Among the investigations, Mantoux positivity was observed in six cases, granulomatous lymphadenitis in 10 cases, and one pleural fluid analysis showed evidence of TB. Of 102 cases, 14 cases were confirmed as TB and one as latent TB infection (LTBI). Among the confirmed cases, cytological study was useful for confirming TB in $71.4 \%$ of cases, Mantoux positivity for five cases of TB (35.7\%), and one case of LTBI. Conclusion: Cervical adenitis and contact history were common among suspected TB children and lymph node cytological study followed by Mantoux test was the useful parameters for confirming TB.
\end{abstract}

Key words: Cervical adenitis, Childhood tuberculosis, Contact survey, Fine-needle aspiration cytology node

I is estimated that nearly 10 million people developed tuberculosis (TB) worldwide in the year 2017, of which nearly 1 million were children [1]. In general, the emphasis is largely focused on the adult disease, whereas the TB in children is relatively neglected mainly due to greater challenges in diagnosis and the comparatively less priority attributed to children by the various TB control programs. As a result of these factors, research and data associated with cases of childhood TB are limited. Childhood TB presents itself as a major challenge due to the method it has been explored till date. Childhood TB is considered as a marker of recent transmission in the community and a pool for future adult cases also [2]. The plan of action to reduce the incidence rate of morbidity and mortality occurring due to childhood TB is to design effective methods of diagnosis at the early stages of the infection, tracing of contacts in addition to designing of an effective treatment regimen.

The non-specific nature of the sign and symptoms, the paucibacillary nature of the disease, and the low specificity of available diagnostic tests contribute vastly to make the cases of childhood TB a daunting task for the clinicians [3]. Differences in the pathophysiology and clinical representation of TB in children make the process of diagnosis more challenging in comparison to adults in addition to non-definitive modes of latent infection and disease are less understood. Following infection, several factors appear to influence the balance of risk between latent TB and progression to active disease. The several factors included are age, nutritional status, vaccination, and immune status [2,3]. Children are at much higher risk of progression into active diseases in comparison to adults. The risk is higher in the cases of infants and children below 2 years to age.

The objective of the present study was to analyze the various symptoms and signs of clinically suspected TB among children as per updated guidelines for pediatric TB 2012 [4] and correlate with the laboratory findings to identify the most useful diagnostic parameters to confirm the clinical diagnosis of TB.

\section{MATERIALS AND METHODS}

This was a retrospective study carried out in a rural tertiary health care hospital for a period of 1 year from January 2017 to 
December 2017. All children under 14 years of age, admitted in the pediatric ward for evaluation of suspected pediatric TB with the following non-specific and organ-specific signs and symptoms were included in our study: History of close contact with an adult diagnosed as TB in the past 2 years, persistent cough ( $>2$ weeks) or fever ( $>2$ weeks), history of poor weight gain or weight loss of $>5 \%$ in the past 3 months, chronic lymphadenitis for $>2$ weeks with either large solitary node of size $>2 \mathrm{~cm}$ or multiple matted or discrete nodes of any size, and at least three or more in the number involving one or both sides of the neck, as well as the incomplete resolution of acute lymphadenitis after a course of antibiotics.

- Pulmonary TB: Respiratory symptoms and lung signs of more than 2 weeks, with or without pleural effusion

- Abdominal TB: Clinical features of chronic pain abdomen, mesenteric adenitis, chronic hepatosplenomegaly, and chronic symptoms involving urinary bladder

- Bone and joint TB: Chronic orthopedic symptoms with suspected radiological findings.

The exclusion criteria consisted of children, who were referred from other with the above signs and symptoms for opinion, where diagnosis other than TB had already been established and who refused to participate. A total of 102 children were studied for suspected TB, during that period and were evaluated by experienced pediatricians for anthropometry (height and weight, nutritional statuses were classified as per the Indian Academy of Pediatrics (IAP) system of classification), baseline hematologic investigations (hemoglobin, total lymphocyte counts, differential leukocyte counts, and erythrocyte sedimentation rate [ESR]), Mantoux/tuberculin skin test (TST) using 1TU, chest X-ray, two early morning samples of either sputum or gastric aspirate smear for acid-fast bacilli (AFB), cytological study of lymph nodes by fine-needle aspiration cytology (FNAC), and other relevant investigations such as USG abdomen and pleural fluid analysis.

Children who were clinically presented with suspected TB symptoms and signs were diagnosed as confirmed $\mathrm{TB}$ with the following criteria: Positive Mantoux test by $>10 \mathrm{~mm}$ in duration, gastric aspirate/sputum smear positive for AFB, chest $\mathrm{X}$-ray showing hilar lymphadenopathy, node FNAC suggestive of granulomatous lymphadenitis, or special investigations like pleural fluid analysis suggestive of TB.

Children evaluated for TB as a part of the contact survey, even without any symptoms and signs, were diagnosed as latent TB infection (LTBI) if they showed positive TST. All demographic, clinical, and investigations data were recorded and analyzed. Data were entered into Microsoft Excel and SPSS was used for data analysis. Data have presented in the form of numbers and percentages. The mean and standard deviation were calculated wherever required.

\section{RESULTS}

A total of 102 children were studied and evaluated for suspected TB during the year 2017, of which 56 were male and 46 were female.
Among the age group, $1(1 \%)$ child was $<1$ year, $19(18.6 \%)$ were between 1 and 3 years, 25 (24.5\%) were between 3.1 and 5 years, $31(30.4 \%)$ were between 5.1 and 10 years, and $26(25.5 \%)$ were above 10 years (Table 1 ).

Out of 102 suspected TB children, 13 showed organ-specific symptoms affecting the abdomen, chest, joint, and parotid glands and the remaining presented with general symptoms. The predominant clinical presentation in the general category of suspected TB children was cervical adenitis $(\mathrm{n}=46 ; 45 \%)$ followed by close contact with an adult patient $(n=34 ; 33.3 \%)$ as shown in Table 2.

Apart from the symptoms documented in the inclusion criteria, children were also presented with other symptoms such as recurrent cough in $23(22.5 \%)$ children, recurrent fever in $9(8.8 \%)$ children, and poor appetite in $25(25 \%)$ children. About $86 \%$ of the children with suspected TB were underweight as per IAP classification, of which $47 \%$ were Grade $1,24.5 \%$ Grade 2, $12.7 \%$ Grade 3 , and $1.9 \%$ had Grade 4 malnutrition.

Among the investigations, Mantoux positivity was observed only in 6 children (5.8\%) and smear for AFB detection was negative in all cases. The common findings noted in chest X-ray

Table 1: Age distribution of suspected and confirmed tuberculosis

\begin{tabular}{lcc}
\hline Age group (year) & Suspected TB (\%) & Confirmed TB (\%) \\
\hline$<1$ & $1(1)$ & - \\
$1-3$ & $19(18.6)$ & $2(14.2)$ \\
$3.1-5$ & $25(24.5)$ & $3(21.4)$ \\
$5.1-10$ & $31(30.4)$ & $4(28.5)$ \\
$10-14$ & $26(25.5)$ & $5(35.7)$ \\
Total & $102(100)$ & $14(100)$ \\
\hline
\end{tabular}

TB: Tuberculosis

Table 2: Clinical features in children evaluated for suspected tuberculosis

\begin{tabular}{llc}
\hline $\begin{array}{l}\text { Symptom } \\
\text { category }\end{array}$ & Symptoms & $\begin{array}{c}\text { Suspected } \\
\text { TB (102) (\%) }\end{array}$ \\
\hline General & Persistent cough & $20(19.6)$ \\
& Persistent fever & $9(8.8)$ \\
& Not thriving & $24(2.5)$ \\
& Weight loss & $3(2.9)$ \\
& Cervical adenitis & $46(45)$ \\
Organ specific & Contact history & $34(33.3)$ \\
& Chest TB, n=1 & \\
& Pleural effusion & 1 \\
& Abdomen TB, n=9 & 3 \\
& Hepatosplenomegaly & 1 \\
& Isolated hepatomegaly & 3 \\
& Chronic pain abdomen & 2 \\
& Chronic dysuria & 1 \\
& TB arthritis, n=2 & 1 \\
& TB knee & \\
TB hip & Recurrent parotid abscess, n=1 & 1 \\
\hline
\end{tabular}

TB: Tuberculosis 
were non-specific in the form of Hilar prominence, increased paracardiac, bronchovascular markings, and few calcific spots in the perihilar regions. Hematological investigations showed anemia in 41 cases (40.2\%), lymphocytosis in 24 cases (23.5\%), and elevated ESR in 40 cases (39.2\%). Of 46 children with cervical lymphadenopathy, the cytological study was possible only for 14 cases (31\%), of which 10 cases $(71.4 \%)$ were reported as granulomatous lymphadenitis (Figure 1) and $4(28.5 \%)$ as nonspecific lymphadenitis. FNAC could not be done for other cases due to its location, small size, the unwillingness of the parent, and poor cooperation of the patient. Pleural fluid analysis from the child who was presented with pleural effusion showed specific findings for $\mathrm{TB}$, but the radiological examination of suspected tuberculous arthritis of two children was not suggestive of TB.

Out of the nine suspected cases of abdominal TB, one case showed isolated hepatomegaly, three children showed hepatosplenomegaly, and two showed mesenteric adenitis and

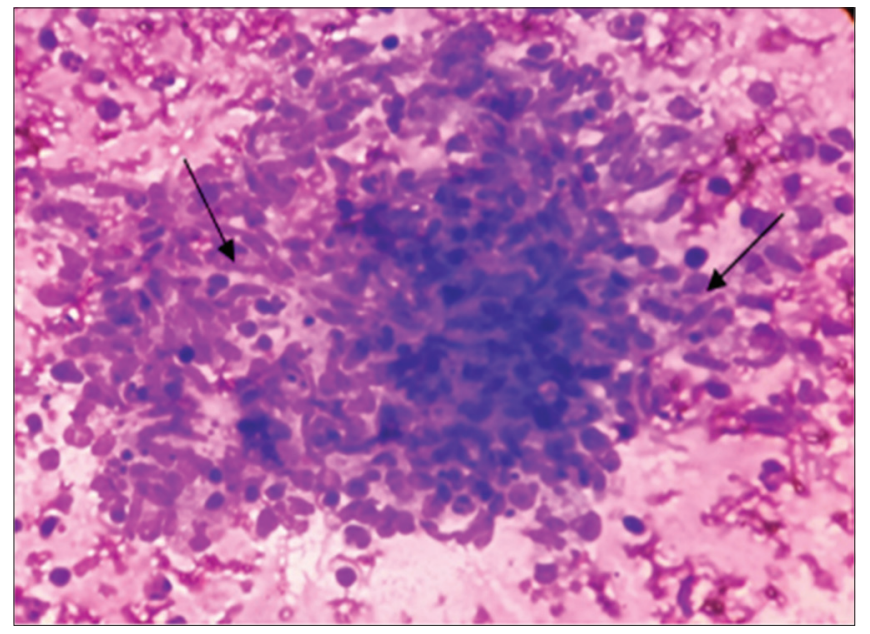

Figure 1: Photomicrograph showing aggregates of elongated slipper-shaped epithelioid cells (arrow) in hemorrhagic background (May-Grunwald-Giemsa $\times 100$ ) there was no strong sonological evidence of TB as shown in Table 3.

Out of the 102 cases evaluated for TB, only 14 children were confirmed positive based on the investigational reports and one case as LTBI. Of the confirmed cases, two were in the age group of 1-3 years, three were between 3.1 and 5 years, 4 were between 5 and 10 years, and 5 were over 10 years of age. Of the 14 cases, nine cases were confirmed based on alone and one with cytological study along with Mantoux positivity. One chest TB was confirmed based on radiological and pleural fluid analysis along with Mantoux positivity. The remaining three cases were confirmed based on the Mantoux positivity along with sonological evidence of hepatosplenomegaly.

\section{DISCUSSION}

Millions of new cases of childhood TB are reported worldwide each year. Pediatric TB has not been given the same priority as adult TB [5]. With the increased risk of developing TB in comparison to the adults, TB in children is also observed in disseminated form with higher mortality rate [6]. In the present study, 45 children (44.1\%) with suspected TB symptoms and 5 children (33\%) of confirmed TB cases were under the age of 5 years, which was similar to the observations from the study conducted by Swaminathan et al. [7] where $34 \%$ of confirmed TB cases were under 5 years. Slight male preponderance in the sex distribution, with a male-to-female ratio of 1.2:1, was observed in the present study which is also supported in other studies $[8,9]$. Despite the various issues associated with non-specific nature of symptoms, signs, and investigations and the emphasis on the various other difficulties faced in the diagnosis of pediatric TB, clinical features continue to remain the foundation of the diagnosis involved in TB [10].

Chronic cervical adenitis (45\%), of which $17 \%$ of cases had solitary nodes, was the most common clinical features observed

Table 3: Investigations in children with suspected TB

\begin{tabular}{|c|c|c|c|c|}
\hline Symptom category & Investigations & Specific findings (n, \%) & Non-specific findings $(\mathrm{n}, \%)$ & Total n (\%) \\
\hline \multirow[t]{8}{*}{ General } & Mantoux & $6(5.8)$ & $96(94.11)$ & $102(100)$ \\
\hline & AFB smear & 0 & $102(100)$ & $102(100)$ \\
\hline & $\mathrm{X}$-ray chest & $1(1)$ & $101(99)$ & $102(100)$ \\
\hline & $\mathrm{CBC}$ & & & \\
\hline & Anemia & $41(40.19)$ & $61(59.8)$ & $102(100)$ \\
\hline & Lymphocytosis & $24(23.53)$ & $78(76.47)$ & $102(100)$ \\
\hline & $\operatorname{ESR}(>20 \mathrm{~mm} / \mathrm{h})$ & $40(39.23)$ & $62(60.78)$ & $102(100)$ \\
\hline & FNAC of lymph nodes & $10(71.4)$ & $4(28.5)$ & $14(100)$ \\
\hline \multirow[t]{8}{*}{ Organ specific } & Chest TB & & & \\
\hline & $\mathrm{X}$-ray chest & $1(100)$ & - & $1(100)$ \\
\hline & Pleural fluid & $1(100)$ & 0 & $1(100)$ \\
\hline & Abdomen TB & & & \\
\hline & Ultrasound & 0 & $9(100)$ & $9(100)$ \\
\hline & Ascitic fluid & 0 & 0 & \\
\hline & TB arthritis & & & \\
\hline & $\mathrm{X}$-ray joint & 0 & $2(100)$ & $2(100)$ \\
\hline
\end{tabular}

ESR: Erythrocyte sedimentation rate, TB: Tuberculosis, CBC: Complete blood count, AFB: Acid-fast bacilli, FNAC: Fine-needle aspiration cytology 
in the present study, the rest of the cases had multiple small to medium-sized matted nodes. A similar observation was made by Swaminathan et al., where $49 \%$ of children with TB had cervical adenitis. The previous history of contact with an adult TB case (34\%) was the second most common reason for suspecting TB in the present study. Out of these, one child was identified with LTBI while the 2 children (5.7\%) were diagnosed with positive TB. In a contact survey study by Jaganath et al., in Uganda, $10 \%$ of cases were diagnosed to have TB and none of the children had serious or disseminated forms of TB [11]. Another study on contact survey from Brazil by Caldeira et al. reported the identification of disease in $13.6 \%$ of cases and LTBI was found in $41 \%$ of child with contacts [12]. In some studies, reverse contact tracing of children with active disease had revealed the presence of 42.5$100 \%$ contact history $[13,14]$. This underscores the importance of contact survey in early diagnosis of pediatric TB.

Contact survey is an important modality for identifying children with TB in developed countries where the burden of TB is low $(<5 \%)$ [15]. However, it is poorly implemented in high burden countries like India, where pediatric TB is estimated to be around $20-40 \%$ of adult TB [16]. The implementation of stringent plans of contact survey in controlling the epidemic of TB in India presents itself with great opportunities. The various plans associated with contact survey such as early diagnosis and treatment, prevention of progression to serious forms of the disease, decrease TB burden in the community, prevention of ongoing transmission, and decrease the future reservoir of adult disease in the community. Contact survey has been strongly recommended by several authors in high burden countries to control the TB epidemic [17].

In the study population, 43 children (42\%) had cough in various forms, whereas Verma et al. and Shrestha et al. reported higher incidence of respiratory complications in $65.3 \%$ and $63.4 \%$ of cases, respectively $[9,10]$. Loss of appetite and fever were observed in $25 \%$ and $17.6 \%$ of the cases in the present study. In a study conducted by Swaminathan et al., it was observed that fever occurred irregularly to a higher number of children (47\%) with confirmed case of TB, whereas in a study by Shrestha et al., it was observed that the occurrence of fever was even higher with $75.6 \%$ of children from the study population with fever.

Another important considered for the development of the disease and plays a key role in the progression to disseminated forms of the disease [18]. In the present study, $86 \%$ of children with suspected TB were malnourished and with $31 \%$ of confirmed cases being underweight. A great degree of variation associated with malnutrition in children with severe diseased conditions has been documented. In a study conducted by Verma et al., of the confirmed cases of TB in children under 5 years of age, the subjects under the category of being considered undernourished were $58.4 \%$, whereas in a study by Seth et al., $58 \%$ of undernutrition rate was observed in the study population [19]. In the present study, the rate of malnutrition was lower; a plausible reason associated with this could earlier presentation of the disease.

Among the study population, only $6 \%$ of children had positive under TST, which was considered to be in the lower range in comparison to other studies while none of them had positive smear for AFB. Shrestha et al. found positive smear in $7.3 \%$ of cases out of 41 confirmed cases while in a study by Swaminathan et al., $0.3 \%$ of the study population had a positive smear out of 2652 suspected cases of TB and 4\% of the 201 confirmed cases. Although confirmation of diagnosis through the aid of bacteriological method was not possible in the present study, FNAC was useful tool that confirmed the presence of TB in the subjects. FNAC helped in clinching the diagnosis in $71 \%$ of the study population subjected to lymph node cytological study, which was also supported by other studies [20].

The radiological finding in the present study was non-specific and not helpful in confirming the diagnosis, except in the child with pleural effusion. Similar difficulties with a radiologic diagnosis have been reported by many researchers. Marias et al. have stated that $90 \%$ of the radiologic abnormalities pass unnoticed in the clinical practice. Delacourt et al. have reported that, of the chest X-ray reported as normal, $60 \%$ had enlarged node on computed tomography of the chest [21]. Du Toit et al. reported wide inter- and intra-observer variability in CXR interpretation in pediatric TB [22]. Jain et al. had shown $31 \%$ discordance in chest X-ray interpretation in his study. Swaminathan et al. in their study found that $58 \%$ of children with proven TB had normal chest X-ray.

The diagnosis of TB was confirmed in $13.7 \%$ of 102 children in the present study, which was high when compared to other studies. Jain et al. obtained $11.6 \%$ of cases of confirmed TB of 223 children and Swaminathan et al. confirmed $7.6 \%$ of the cases of TB, of 2625 children evaluated for suspected TB. An astounding $71.4 \%$ of the confirmed cases of $\mathrm{TB}$ in the present study were diagnosed by FNAC. The present study faced certain major limitation in terms of the study design; a major drawback was the small size of the study population, a large sample size could have provided more conclusive outcomes that might have been more useful. Another demerit of the present study was the absence of histopathological evaluation to confirm TB.

\section{CONCLUSION}

The diagnosis of TB in children remains a challenging one, due to non-specific clinical features and investigations and a strict contact survey was useful for picking up the early cases of TB. Cervical adenopathy was a common presentation in children with suspected TB and FNAC was a most useful early diagnostic tool for confirming suspected TB.

\section{ACKNOWLEDGMENT}

The authors would like to thank Dr. T. Ramesh, Managing director of MAPIMS for all the support provided by the institution in conducting this study.

\section{REFERENCES}

1. World Health Organization. Global Tuberculosis Report 2018. Geneva: World Health Organization; 2018. 
2. Piccini P, Chiappini E, Tortoli E, De Martino M, Galli L. Clinical peculiarities of tuberculosis. BMC Infect Dis 2014;14:S4.

3. Marais BJ, Graham SM, Cotton MF, Beyers N. Diagnostic and management challenges for childhood tuberculosis in the era of HIV. J Infect Dis 2007;196:S76-85.

4. Kumar A, Gupta D, Nagaraja SB, Singh V, Sethi GR, Prasad J. Updated national guidelines for pediatric tuberculosis in India 2012. Indian Pediatr 2013;50:301-6.

5. Swaminathan S. Evidence-based treatment of tuberculosis for children: The unfinished agenda. Indian Pediatr 2010;47:39-40.

6. Nelson LJ, Wells CD. Global epidemiology of childhood tuberculosis. Int J Tuberc Lung Dis 2004;8:636-64.

7. Swaminathan S, Datta M, Radhamani MP, Mathew S, Reetha AM, Rajajee S, et al. A profile of bacteriologically confirmed pulmonary tuberculosis in children. Indian Pediatr 2008;45:743-7.

8. Jain SK, Ordonez A, Kinikar A. Pediatric tuberculosis in young children in India: A prospective study. BioMed Res Int 2013;2013:1-7.

9. Verma J, Ahirwal K, Patel U, Shingwekar AG, Sharma S. Clinical profile of tuberculosis in children up to 5 years of age. Pediatr Rev Int J Pediatr Res 2014;1:10-7.

10. Shrestha S, Bichha RP, Sharma A. Clinical profile of tuberculosis in children. Nepal Med Coll J 2011;13:119-22.

11. Jaganath D, Zalwango S, Okwareetal B. Contact investigation for active tuberculosis among child contacts in Uganda. Clin Infect Dis 2013;57:1685-92.

12. Caldeira ZM, Sant'Anna CC, Aidé MA. Tuberculosis contact tracing among children and adolescents, Brazil. Rev Saude Publica 2004;38:1-6.

13. Cho YH, Ho TS, Wang SM, Shen CF, Chuang PK, Liu CC. Childhood tuberculosis in Southern Taiwan, with emphasis on central nervous system complications. J Microbiol Immunol Infect 2014;47:503-11.

14. Bolursaz MR, Khalilzadeh S, Baghaie N, Mehrian P, Ghafaripoor H, Aghahosseini $\mathrm{F}$, et al. Clinical and radiographic findings of pulmonary tuberculosis in infants. J Compr Pediatr 2013;3:170-4.

15. Starke JR, Taylor-Watts KT. Tuberculosis in the pediatric population of Houston, Texas. Pediatrics 1989;84:28-35.

16. Newton SM, Brent AJ, Anderson S, Whittaker E, Kampmann B. Paediatric tuberculosis. Lancet Infect Dis 2008;8:498-510.

17. Marais BJ, Gie RP, Schaaf HS, Hesseling AC,Obihara CC, Nelson LJ, et al. The clinical epidemiology of childhood pulmonary tuberculosis: A critical review of literature from the pre-chemotherapy era. Int J Tuberc Lung Dis 2004;8:278-85.

18. Jaganath D, Mupere E. Childhood tuberculosis and malnutrition. J Infect Dis 2012;206:1809-15.

19. Seth V, Singhal PK, Semwal OP, Kabra SK, Jain Y. Childhood tuberculosis in a referral centre: Clinical profile and risk factors. Indian Pediatr 1993;30:479-85.

20. Wright CA, Hesseling AC, Bamford C, Burgess SM, Warren R, Marais BJ. Fine-needle aspiration biopsy: A first-line diagnostic procedure in paediatric tuberculosis suspects with peripheral lymphadenopathy ? Int J Tuberc Lung Dis 2009;13:1373-9.

21. Delacourt C, Mani TM, Bonnerot V, De Blic J, Sayerg N, Lallemand D, et al. Computed tomography with normal chest radiograph in tuberculous infection. Arch Dis Child 1993;69:430-2.

22. Du Toit $G$, Swingler G, Iloni K. Observer variation in detecting lymphadenopathy on chest radiography. Int J Tuberc Lung Dis 2002;6:814-7.

Funding: None; Conflict of Interest: None Stated.

How to cite this article: Kalyanaraman P, Sumathi S. Clinicopathological profile of children evaluated for suspected tuberculosis in a rural medical college hospital. Indian J Child Health. 2019; 6(7):365-369.

Doi: 10.32677/IJCH.2019.v06.i07.009 